\title{
Neurological Involvement in Systemic Lupus Erythematosus (SLE): Our Recent Experience
}

\author{
B. S. Kane ${ }^{1 *}$, M. Dieng1, B. C. Fall1, M. Sow ${ }^{1}$, A. C. Ndao¹, B. Djiba1, M. Niasse², N. Diagne1, A. Faye1, \\ S. Ndongo' ${ }^{1}$, A. Pouye ${ }^{1}$
}

\begin{abstract}
${ }^{1}$ Department of Internal Medicine, Le Dantec Teaching Hospital; Cheikh Anta DIOP University of DAKAR, Dakar, Senegal ${ }^{2}$ Department of Rheumatology, Le Dantec Teaching Hospital; Cheikh Anta DIOP University of DAKAR, Dakar, Senegal Email: *baidysy.kane@ucad.edu.sn
\end{abstract}

How to cite this paper: Kane, B.S., Dieng, M., Fall, B.C., Sow, M., Ndao, A.C., Djiba, B., Niasse, M., Diagne, N., Faye, A., Ndongo, S. and Pouye, A. (2019) Neurological Involvement in Systemic Lupus Erythematosus (SLE): Our Recent Experience. Open Journal of Rheumatology and Autoimmune Diseases, 9, 25-34.

https://doi.org/10.4236/ojra.2019.92003

Received: February 25, 2019

Accepted: May 3, 2019

Published: May 6, 2019

Copyright $\odot 2019$ by author(s) and Scientific Research Publishing Inc. This work is licensed under the Creative Commons Attribution International License (CC BY 4.0).

http://creativecommons.org/licenses/by/4.0/

\begin{abstract}
Introduction: The central, psychiatric and peripheral neurological manifestations of lupus are among the most severe visceral disorders and are grouped under the general term of "neuro-psychiatric systemic lupus erythematosus" (NPSLE). We conducted a cross-sectional observational study within our Department of Internal Medicine aimed at describing the clinical and evolutionary aspects of central neurological disorders of SLE, excluding lupus myelopathy. Patients and Methods: This was a retrospective and observational cross-sectional study carried out from 1 January 2015 to 31 October 2017, in the Department of Internal Medicine of Aristide le Dantec University Hospital in Dakar (Senegal). All patients hospitalized during this period who met the 1997 ACR classification criteria of SLE and who presented with a central neuropsychiatric syndrome attributable to SLE (as defined by ACR 1999) were included. Patients with isolated headache, acute myelitis or secondary neurological involvement attributable to a toxic, metabolic, infectious or tumour-related cause were excluded from our study. Results: During the study period, 10 patients with neuropsychiatric lupus involvement were treated at our institution, including 9 women and 1 man; the median age was 29 years (20 - 55 years). Neurological involvement occurred during the course of lupus evolution in $9 / 10$ cases. The median time to SLE evolution was 18 months (0 - 60 months). Neuropsychiatric syndromes as defined by the 1999 ACR were commonly associated and more than half of our patients had multiple neuropsychiatric syndromes. There were 5 cases of confusion syndrome and coma, 4 cases of seizure, 3 cases of psychosis, 2 cases of acute cerebrovascular disease and 1 case of aseptic meningitis. Among the extra-neurological manifestations of SLE, haematological and dermatological involvements were common. Renal involvement affected half of the patients. The other manifestations were: polyarthritis in 3 patients, se-
\end{abstract}


rositis in 2 patients, 5 cases of fever, 4 cases of deterioration of the general state, and one isolated case of ophthalmological involvement. Therapeutically, 8 patients received a bolus of methylprednisolone and 3 patients received a bolus of cyclophosphamide. Oral corticosteroids and hydroxychloroquine were administered to all patients, and azathioprine was administered in 2 patients. The evolution was favorable in 4 patients, other 2 patients maintained neurological sequelae and 2 patients were transferred to intensive care. Death was recorded in 4 patients. Conclusion: Neuropsychiatric manifestations of lupus are rare and sometimes severe, potentially life-threatening. In our patients, we have identified some of the most severe neurological syndromes according to the ACR nomenclature. The neurological involvement is exceptionally revealing, as these syndromes are often associated and integrated into a systemic context of lupus. The evolution is rapidly unfavorable and requires early diagnosis and optimal management.

\section{Keywords}

Systemic Lupus Erythematosus, Lupus Vasculitis, Central Nervous System, Africa South of the Sahara

\section{Introduction}

Systemic lupus erythematosus (SLE) is a systemic autoimmune disease of unknown cause [1] [2]. It is characterized by heterogeneous, multisystem involvement with protean manifestations and biologically by multiple anomalies dominated by the presence of antinuclear antibodies, directed against the constituents of the nucleus. It represents a prototypic systemic autoimmune disease [3] [4].

The central, psychiatric and peripheral neurological manifestations of lupus are among the most severe visceral disorders and are grouped under the general term of "neuro-psychiatric systemic lupus erythematosus" (NPSLE) [5]. In 1999, an expert panel of the American College of Rheumatology (ACR) defined 19 clinical situations that are observed with NPSLE. This classification includes a wide range of syndromes and distinguishes between central and peripheral manifestations of NPSLE (12 in the central nervous system (CNS) and 7 in the peripheral nervous system) [6].

The overall prevalence of these events varies significantly between studies, ranging from $20 \%$ to $97 \%$ [7] [8] [9]. In a recent meta-analysis identifying all of the studies based on the ACR criteria and identifying the most robust from a methodological point of view, the overall prevalence of neuropsychiatric manifestations was estimated at 56\% [10]. These are extremely heterogeneous conditions and, in terms of diagnosis, the clinician is constantly confronted with two difficulties: on the one hand, the risk of "under diagnosing" authentic NPSLE which may delay appropriate management, and on the other hand, the risk of "over-diagnosing" NPSLE [11]. In addition, the neurological syndromes 
identified in the ACR nomenclature are not specific to SLE and do not constitute diagnostic criteria. Diagnostic and therapeutic management remains a challenge for the clinician [5].

Specific studies on the neurological manifestations of SLE have been reported exceptionally in sub-Saharan Africa [12]. Thus, we conducted a cross-sectional observational study within our Department of Internal Medicine aimed at describing the clinical and evolutionary aspects of central neurological disorders of SLE, excluding lupus myelopathy.

\section{Patients and Methods}

This was a retrospective and observational cross-sectional study carried out from 1 January 2015 to 31 October 2017, in the department of Internal Medicine of Aristide le Dantec University Hospital in Dakar (Senegal). All patients hospitalized during this period who met the 1997 ACR classification criteria of SLE and who presented with a central neuropsychiatric syndrome attributable to SLE (as defined by ACR 1999) were included. Patients with isolated headache, acute myelitis or secondary neurological involvement attributable to a toxic, metabolic, infectious or tumor-related cause were excluded from our study. The approval of the local ethics committee was required.

From all patients included in the study, we collected:

- epidemiological data: age and sex;

- clinical data: duration of lupus, the chronology of the appearance of neurological involvement in relation to the diagnosis of the disease, the neurological syndrome, the extra-neurological visceral involvement of SLE and the activity of the disease assessed by SLEDAI (Systemic Lupus Erythematosus Disease Activity Index)-2K [13];

- biological data: complete blood count, C-reactive protein, cyto-bacteriological examination of the cerebrospinal fluid and immunological assays such as antibodies to anti-nuclear (AAN), antibodies to (double-stranded) dsDNA, antibodies to extractable nuclear antigens (anti-ENA) and anti-phospholipid (aPL) antibodies;

- encephalic imaging: magnetic resonance imaging (MRI) and cerebral computed tomography (CT);

- Data on disease history and progression: favorable or unfavorable (based on the development of neurological sequelae or the occurrence of death).

All data were captured and analyzed using SPSS 20 software. Quantitative variables are expressed as median values with extremes and qualitative variables as numbers and percentages.

\section{Results}

During the study period, 10 patients with neuropsychiatric lupus involvement were treated at our institution, including 9 women and 1 man; the median age was 29 years (20 - 55 years). Neurological involvement occurred during the 
course of lupus evolution in $9 / 10$ cases. The median time to SLE evolution was 18 months (0 - 60 months). Neuropsychiatric syndromes as defined by the 1999 ACR were commonly associated. In fact, $6 / 10$ patients had at least 02 neuropsychiatric syndromes. There were 5 cases of confusion syndrome and coma, 4 cases of seizure, 3 cases of psychosis, 2 cases of focal deficit syndrome related to a cerebrovascular accident and 1 case of meningeal syndrome. We summarized the neurological manifestations in our patients in Table 1. Other neurological manifestations outside of the central neuropsychiatric syndromes according to ACR classification in our patients, were 01 case of extrapyramidal symptoms and one case of mononeuropathy/multiplex.

Among the extra-neurological manifestations of SLE, haematological and dermatological involvements were consistent. Renal involvement affected half of the patients with stage III + V lupus nephropathy (two cases) and isolated cases at stages III, IV and V. The other manifestations were: polyarthritis in 3 patients, serositis in 2 patients, 5 cases of fever, 4 cases of deterioration of the general state, and one isolated case of ophthalmological involvement (occlusion of the central vein of the retina without antiphospholipid syndrome ) We summarized the systemic manifestations of Lupus disease in our patients in Table 2.

Biologically, anaemia, lymphopenia and anacceleratederythrocyte sedimentation rate (ESR) were common. Median ESR was $103 \mathrm{~mm}(85$ - 143) in the first hour, median C-reactive protein was $19 \mathrm{mg} / \mathrm{l}(0$ - 48) and the median SLEDAI-2K score was 19 (12 - 23).

Table 1. Neuropsychiatric syndromes in our patients.

\begin{tabular}{cc}
\hline Central nervous system & Number of cases (\%) \\
\hline Acute confusional state and coma & $05(50 \%)$ \\
Seizure disorder & $04(40 \%)$ \\
Psychosis & $03(30 \%)$ \\
Cerebrovascular disease & $02(20 \%)$ \\
Aseptic meningitis & $01(10 \%)$ \\
\hline
\end{tabular}

Table 2. Visceral involvements associated to NPSLE in our study.

\begin{tabular}{cc}
\hline Manifestations of SLE & Number of cases (\%) \\
\hline Hematological disorder (Cytopenia) & $10(100 \%)$ \\
Mucocutaneous lesions & $10(100 \%)$ \\
Lupus Nephritis & $05(50 \%)$ \\
Fever & $05(50 \%)$ \\
General state dysfunction & $04(40 \%)$ \\
Arthritis & $03(30 \%)$ \\
Serositis & $02(20 \%)$ \\
Ophthalmological disorder & $01(10 \%)$ \\
\hline
\end{tabular}


A lumbar puncture performed in 6 patients indicated pathological CSF in half of the cases (with high protein levels in the CSF), pleiocytosis and without infection (assessed by direct examination after Gram staining, cultures on conventional and specific media).

Brain imaging performed in 7 patients and was pathological in 5 cases.

Immunologically, antinuclear antibodies were positive in all cases where they were assessed. The following antibodies were screened in the following proportions: anti-dsDNA (2/2) and anti-Sm (3/4). Therapeutically, 05 patients received a bolus of methylprednisolone alone and 3 patients received both a bolus of methylprednisolone and cyclophosphamide. Oral corticosteroids and hydroxychloroquine were administered to all patients, and azathioprine was administered in 2 patients. The evolution was favorable in 4 patients, another 2 patients maintained neurological sequelae and death was recorded in 4 patients. 02/10 patients were transferred to intensive care. These patients didn't received a bolus of methylprednisolone in this department.

\section{Discussion}

We performed this study specifically dedicated to NPSLE. This visceral involvement of lupus has been the subject of limited studies in the literature in sub-Saharan Africa [12].

The overall prevalence of NPSLE is highly variable, i.e. from $20 \%$ to $97 \%$ according to previous studies, despite the consensus attempt of the ACR working group. Through a recent meta-analysis, NPSLE has been found to occur at a rate of 56\% [10]. Due to methodological limitations related to patient recruitment, i.e. exclusively from a hospital, no prevalence data could be deduced from our work. Due also to our retrospective design and the limitations of financial resources we didn't perform cerebral imaging and the determination of antiphospholipid antibodies in all patients.

Clinically, we have identified among our patients the most severe neurological syndromes according to the nomenclature of the ACR. They were most often associated and integrated into the systemic context of lupus. In the literature, mild neurological conditions such as headache or mood disorders were the most common symptoms in an international prospective cohort study on lupus [14] [15]. These neurologic syndromes were attributable to other causes apart from SLE and were not associated with disease activity [14] [15]. Besides, major neuropsychiatric manifestations such as those encountered in our study are rare. Kampylafka et al. reported these symptoms (including acute confusion, seizures, aseptic meningitis, chorea, cerebrovascular accidents, myelopathy and psychosis) in $16 / 370$ patients (4.3\%) after a prospective follow-up period of three years [16]. This clinical presentation in our patients could be related to a recruitment bias of the most severe patients in the hospitalisation sector, without excluding the influence of genetic factors in patients of African ethnicity.

In order of frequency, the manifestations most often encountered in the lite- 
rature are headache $(28 \%)$, thymic disorders $(21 \%)$, cognitive disorders $(20 \%)$, seizures (10\%), cerebrovascular accidents (8\%) and anxiety (6\%) [10]. In our study, the predominant neuropsychiatric disorders were confusion syndrome and coma, seizures and psychoses. Disorders of consciousness, i.e. confusion syndrome and coma, are often symptoms rather than real entities, and attributing these symptoms to lupus remains difficult. Although they predominated in our study, the exclusive responsibility of SLE is exceptional in most studies. In the paediatric series, the prevalence of this syndrome was 35\%, also it can be found at elderly patients [17] [18] [19].

All forms of seizures can occur during SLE: generalized seizures (tonic-clonic, atonic or even absence type, or purely myoclonic) have been described, but simple partial seizures (without alertness disorder) or complexes (with a disturbance in alertness), secondarily generalized or not, are the most frequent in most series. If prescribing precedes the diagnosis of SLE, the hypothesis of lupus induced by anticonvulsants must be raised [20]. About $5 \%$ to $16 \%$ of adult SLE patients and $3 \%$ to $51 \%$ of children have at least one seizure disorder, while the prevalence of seizures is less than $1 \%$ in the general population [5] [16]. The associated risk factors are all found in our study with the exception of the presence of a PL. These include systemic lupus, the presence of anti-Sm antibodies, the presence of other neurological manifestations, young age and ethnic origin (African-American and Hispanic) [21].

Psychosis was diagnosed in 3 of our patients. It has a prevalence of $8 \%$ and is rarely isolated, often associated with other neuropsychiatric disorders. The central neurological manifestation least found in our study was meningeal involvement. Indeed, aseptic meningitis is exceptional in lupus and affects fewer than $2 \%$ of patients [22]. They often pose a differential diagnosis problem with drug-induced aseptic meningitis, mainly due to treatment with non-steroidal anti-inflammatory drugs and trimethoprim-cotrimoxazole [23]. In our context, viral meningitis or certain bacteria that require specific culture techniques (such as Salmonella typhi, Mycobacterium tuberculosis, Listeria monocytogenes or Cryptococcus neoformans) that appear to be overrepresented in lupus should be mentioned [22] [24] [25] [26]. Two cases of stroke were noted in our study, including one case of haemorrhagic stroke due to the rupture of an aneurysm. Strokes occur in $2 \%$ to $24 \%$ of patients with lupus according to published studies, or in $5 \%$ to $10 \%$ according to the experts of the European League against Rheumatism (EULAR) [21]. Bleeding haemorrhagic events or meningeal haemorrhage may be promoted by thrombocytopenia, anticoagulant therapy or arterial hypertension.

In the absence of a gold standard, the diagnosis of a NPSLE remains difficult. The identification of a neurological syndrome attributable to lupus in accordance with the ACR nomenclature must have the chronology of its installation analyzed. Indeed, it occurs most often at the beginning of the disease, and in $40 \%$ of cases during follow-up, and is rarely revealing [21] [27] [28]. The exclusion of a secondary cause, especially related to toxins, drugs, metabolic alterations and especially infections in our context, was a decisive step in our ap- 
proach. Lupus was active (according to SLEDAI) in all our patients with associated systemic manifestations (Table 2). Biologically, the combination of cytopenia, accelerated ESR and low CRP was consistent among all our patients and could be an inexpensive referral in our context. If MRI remains the gold standard of neurolupus imaging, more accessible CT has been crucial in our patients with neurological focal syndrome (Figure 1).

However, it should be remembered that the association of other systemic manifestations with neuropsychiatric manifestations should not systematically rule out an infectious cause in patients already on immunosuppressive therapy or whose immunosuppressive treatments have recently been increased. Beyond this, it should be noted that, out of 10 patients, 6 had problems with their conventional Disease Modifying Anti-Rheumatic Drugs (DMARD). In our study, the NPSLE occurred in the initial diagnosis of SLE in 02 patients. We have identified in the other patients, trigger factors such as voluntary therapeutic interruption (4 patients) or inappropriate previous treatment ( 2 cases).

The therapeutic management of patients was guided by the severity of the NPSLE, in our patients. Symptomatic therapy (anticonvulsants or antipsychotics) have been considered (when appropriate) and almost all patients received a therapy targeting immune after exclusion of non-SLE related causes. Thus, all patients except those transferred to intensive care received a bolus of methylprednisolone, and 3 patients were treated with both bolus of cyclophosphamide and methylprednisolone. Since NPSLE is a rare manifestation of the disease, its treatment is empirical. There has been only one comparative trial showing the superiority of a methylprednisolone-cyclophosphamide bolus compared to a methylprednisolone bolus alone in severe forms of NPSLE [29]. Corticosteroids and

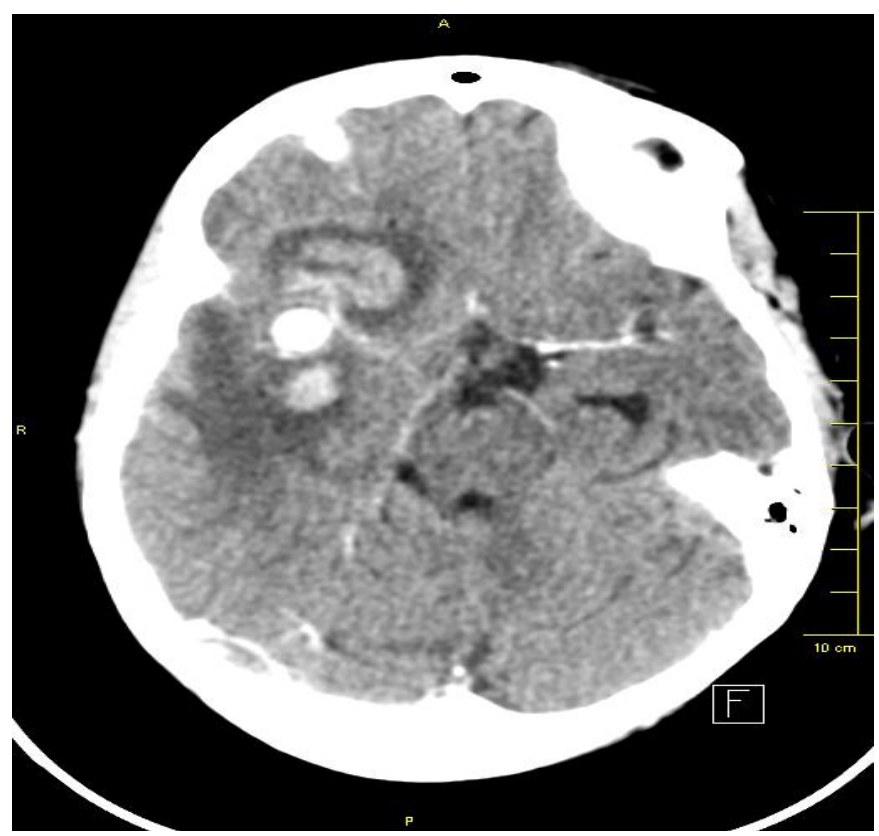

Figure 1. Axial computer tomography. Haemorrhagic stroke by aneurysm rupture in one of our patient. 
immunosuppressive therapy are recommended for manifestations related to an inflammatory and/or autoimmune process: aseptic meningitis, acute confusion syndrome, myelitis, psychosis, polyneuropathy and cranial nerve involvement (level of evidence 1A according to EULAR experts) [17]. Apart from these situations, EULAR experts recommend the use of immunosuppressants according to the activity of the disease outside of NPSLE [17].

Neurological involvement has been associated with a poor prognosis, leading to sequelae and disability, impaired quality of life or death [30]. The evolution was unfavourable for 6 of our patients (with 2 neurological sequelae and 4 deaths).

\section{Conclusions}

Neuropsychiatric manifestations of lupus are rare and sometimes severe, potentially life-threatening. In our patients, we have identified some of the most severe neurological syndromes according to the ACR nomenclature. The neurological involvement is exceptionally revealing, as these syndromes are often associated and integrated into a systemic context of lupus. The evolution is rapidly unfavorable and requires early diagnosis and optimal management.

This diagnosis remains difficult in the absence of reliable diagnostic markers for each type of clinical manifestation. It must always be based on a set of chronological, clinical and paraclinical arguments. Nevertheless, the sensitivity and specificity of the various tools at our disposal vary enormously from one manifestation to another. Indeed, it is unlikely that a diagnostic tool alone will be applicable to all manifestations of NPSLE. It is therefore necessary to develop tools applicable to each type of neurological or psychiatric manifestation associated with lupus.

\section{Conflicts of Interest}

The authors declare no conflicts of interest regarding the publication of this paper.

\section{References}

[1] Nossent, J.C. and Swaak, A.J. (1991) Prevalence and Significance of Haematological Abnormalities in Patients with Systemic Lupus Erythematosus. The Quarterly Journal of Medicine, 80, 605-612.

[2] Keeling, D.M. and Isenberg, D.A. (1993) Haematological Manifestations of Systemic Lupus Erythematosus. Blood Reviews, 7, 199-207. https://doi.org/10.1016/0268-960X(93)90006-P

[3] Van Dam, A.P. (1991) Diagnosis and Pathogenesis of CNS Lupus. Rheumatology International, 11, 1-11. https://doi.org/10.1007/BF00290244

[4] Carbotte, R.M., Denburg, S.D. and Denburg, J.A. (1986) Prevalence of Cognitive Impairment in Systemic Lupus Erythematosus. The Journal of Nervous and Mental Disease, 174, 357-364. https://doi.org/10.1097/00005053-198606000-00008

[5] Schwartz, N., Stock, A.D. and Putterman, C. (2019) Neuropsychiatric Lupus: New Mechanistic Insights and Future Treatment Directions. Nature Reviews Rheuma- 
tology, 15, 137-152. https://doi.org/10.1038/s41584-018-0156-8

[6] (1999) The American College of Rheumatology Nomenclature and Case Definitions for Neuropsychiatric Lupus Syndromes. Arthritis \& Rheumatology, 42, 599-608. https://doi.org/10.1002/1529-0131(199904)42:4<599::AID-ANR2>3.0.CO;2-F

[7] Lefèvre, G., Zéphir, H., Warembourg, F., Michelin, E., et al. (2012) Neuropsychiatric Systemic Lupus Erythematosus (1st Part). Cases Definitions and Diagnosis and Treatment of Central Nervous System and Psychiatric Manifestations of Systemic Lupus Erythematosus. La Revue de Médecine Interne, 33, 491-502. https://doi.org/10.1016/j.revmed.2012.03.356

[8] Ainiala, H., Loukkola, J., Peltola, J., Korpela, M. and Hietaharju, A. (2001) The Prevalence of Neuropsychiatric Syndromes in Systemic Lupus Erythematosus. Neurology, 57, 496-500. https://doi.org/10.1212/WNL.57.3.496

[9] Bertsias, G.K. and Boumpas, D.T. (2010) Pathogenesis, Diagnosis and Management of Neuropsychiatric SLE Manifestations. Nature Reviews Rheumatology, 6, 358-367. https://doi.org/10.1038/nrrheum.2010.62

[10] Brey, R.L., Holliday, S.L., Saklad, A.R., Navarrete, M.G., Hermosillo-Romo, D., Stallworth, C.L., et al. (2002) Neuropsychiatric Syndromes in Lupus: Prevalence Using Standardized Definitions. Neurology, 58, 1214-1220.

https://doi.org/10.1212/WNL.58.8.1214

[11] Lefèvre, G., Zéphir, H., Michelin, E., Semah, F., et al. (2012) Neuropsychiatric Systemic Lupus Erythematosus (2nd Part). Diagnostic and Treatment Tools in Psychiatric or Central Nervous System Manifestations in Systemic Lupus Erythematosus. La Revue de Médecine Interne, 33, 503-513. https://doi.org/10.1016/j.revmed.2012.03.354

[12] Adelowo, O.O., Oguntona, A.S. and Ojo, O. (2009) Neuropsychiatric Systemic Lupus Erythematosus among Nigerians. African Journal of Medicine and Medical Sciences, 38, 33-38.

[13] Rao, V. and Gordon, C. (2014) Advances in the Assessment of Lupus Disease Activity and Damage. Current Opinion in Rheumatology, 26, 510-519. https://doi.org/10.1097/BOR.0000000000000085

[14] Hanly, J.G., Urowitz, M.B., O’Keeffe, A.G., Gordon, C., Bae, S.C., Sanchez-Guerrero, J., et al. (2013) Headache in Systemic Lupus Erythematosus: Results from a Prospective, International Inception Cohort Study. Arthritis \& Rheumatology, 65, 2887-2897. https://doi.org/10.1002/art.38106

[15] Hanly, J.G., Su, L., Urowitz, M.B., Romero-Diaz, J., Gordon, C., Bae, S.C., et al. (2015) Mood Disorders in Systemic Lupus Erythematosus: Results from an International Inception Cohort Study. Arthritis \& Rheumatology, 67, 1837-1847.

[16] Kampylafka, E.I., Alexopoulos, H., Kosmidis, M.L., Panagiotakos, D.B., Vlachoyiannopoulos, P.G., Dalakas, M.C., et al. (2013) Incidence and Prevalence of Major Central Nervous System Involvement in Systemic Lupus Erythematosus: A 3-Year Prospective Study of 370 Patients. PLOS ONE, 8, e55843. https://doi.org/10.1371/journal.pone.0055843

[17] Sibbitt Jr., W.L., Brandt, J.R., Johnson, C.R., Maldonado, M.E., Patel, S.R., Ford, C.C., et al. (2002) The Incidence and Prevalence of Neuropsychiatric Syndromes in Pediatric Onset Systemic Lupus Erythematosus. The Journal of Rheumatology, 29, 1536-1542.

[18] Dennis, M.S., Byrne, E.J., Hopkinson, N. and Bendall, P. (1992) Neuropsychiatric Systemic Lupus Erythematosus in Elderly People: A Case Series. Journal of Neurology, Neurosurgery, and Psychiatry, 55, 1157-1161. 
https://doi.org/10.1136/jnnp.55.12.1157

[19] Heckman, G.A., Papaioannou, A., Adachi, J.D. and Denburg, J.A. (2001) Systemic Lupus Erythematosus Presenting as Subacute Delirium in an 82-Year-Old Woman. Journal of the American Geriatrics Society, 49, 458-461. https://doi.org/10.1046/j.1532-5415.2001.49093.x

[20] Mikdashi, J., Krumholz, A. and Handwerger, B. (2005) Factors at Diagnosis Predict Subsequent Occurrence of Seizures in Systemic Lupus Erythematosus. Neurology, 64, 2102-2107. https://doi.org/10.1212/01.WNL.0000165959.98370.D5

[21] Bertsias, G.K., Ioannidis, J.P., Aringer, M., Bollen, E., Bombardieri, S., Bruce, I.N., et al. (2010) EULAR Recommendations for the Management of Systemic Lupus Erythematosus with Neuropsychiatric Manifestations: Report of a Task Force of the EULAR Standing Committee for Clinical Affairs. Annals of the Rheumatic Diseases, 69, 2074-2082. https://doi.org/10.1136/ard.2010.130476

[22] Baizabal-Carvallo, J.F., Delgadillo-Marquez, G., Estanol, B. and Garcia-Ramos, G. (2009) Clinical Characteristics and Outcomes of the Meningitides in Systemic Lupus Erythematosus. European Neurology, 61, 143-148.

https://doi.org/10.1159/000186504

[23] Moris, G. and Garcia-Monco, J.C. (1999) The Challenge of Drug-Induced Aseptic Meningitis. Archives of Internal Medicine, 159, 1185-1194.

https://doi.org/10.1001/archinte.159.11.1185

[24] Gerona, J.G. and Navarra, S.V. (2009) Salmonella Infections in Patients with Systemic Lupus Erythematosus: A Case Series. International Journal of Rheumatic Diseases, 12, 319-323. https://doi.org/10.1111/j.1756-185X.2009.01440.x

[25] Gonzalez, L.A., Vasquez, G., Restrepo, J.P., Velasquez, M. and Ramirez, L.A. (2010) Cryptococcosis in Systemic Lupus Erythematosus: A Series of Six Cases. Lupus, 19, 639-645. https://doi.org/10.1177/0961203309356458

[26] Tobon, G.J., Serna, M.J. and Canas, C.A. (2010) Listeria Monocytogenes Infection in Patients with Systemic Lupus Erythematosus. Clinical Rheumatology, 32, S25-S27. https://doi.org/10.1007/s10067-010-1416-4

[27] Ainiala, H., Hietaharju, A., Loukkola, J., Peltola, J., Korpela, M., Metsanoja, R., et al. (2001) Validity of the New American College of Rheumatology Criteria for Neuropsychiatric Lupus Syndromes: A Population-Based Evaluation. Arthritis \& Rheumatology, 45, 419-423. https://doi.org/10.1002/1529-0131(200110)45:5<419::AID-ART360>3.0.CO;2-X

[28] Unterman, A., Nolte, J.E., Boaz, M., Abady, M., Shoenfeld, Y. and Zandman-Goddard, G. (2011) Neuropsychiatric Syndromes in Systemic Lupus Erythematosus: A Metaanalysis. Seminars in Arthritis and Rheumatism, 41, 1-11. https://doi.org/10.1016/j.semarthrit.2010.08.001

[29] Barile-Fabris, L., Ariza-Andraca, R., Olguin-Ortega, L., Jara, L.J., Fraga-Mouret, A., Miranda-Limon, J.M., et al. (2005) Controlled Clinical Trial of IV Cyclophosphamide versus IV Methylprednisolone in Severe Neurological Manifestations in Systemic Lupus Erythematosus. Annals of the Rheumatic Diseases, 64, 620-625. https://doi.org/10.1136/ard.2004.025528

[30] Hanly, J.G., Urowitz, M.B., Su, L., Bae, S.C., Gordon, C., Wallace, D.J., et al. (2010) Prospective Analysis of Neuropsychiatric Events in an International Disease Inception Cohort of Patients with Systemic Lupus Erythematosus. Annals of the Rheumatic Diseases, 69, 529-535. https://doi.org/10.1136/ard.2008.106351 